\title{
Tabularia
}

TABULARIA Sources écrites des mondes normands médiévaux Guillaume de Volpiano : Fécamp et l'histoire normande | 2002

\section{Fécamp et les rois anglo-normands}

Fécamp and the anglo-norman kings

Judith Ann Green

\section{(2) OpenEdition}

Journals

Édition électronique

URL : http://journals.openedition.org/tabularia/1704

DOI : 10.4000/tabularia.1704

ISSN : 1630-7364

Éditeur :

CRAHAM - Centre Michel de Boüard, Presses universitaires de Caen

Référence électronique

Judith Ann Green, «Fécamp et les rois anglo-normands », Tabularia [En ligne], Guillaume de Volpiano : Fécamp et l'histoire normande, mis en ligne le 10 juillet 2002, consulté le 21 avril 2019. URL : http:// journals.openedition.org/tabularia/1704; DOI : 10.4000/tabularia.1704 


\title{
Fécamp et les rois anglo-normands Fécamp and the anglo-norman kings
}

\author{
Judith Ann GREEN \\ School of Modern History, \\ The Queen's University of Belfast, \\ Belfast BT7 1NN \\ Northern Ireland \\ j.green@qub.ac.uk
}

Résumé :

Les expériences de l'abbaye de Fécamp peuvent servir d'exemple des relations entre les rois/ ducs et les grands ecclésiastiques. L'abbaye avait déjà acquis des possessions en Angleterre avant 1066 et avait aidé Guillaume le Conquérant pour son expédition. À Pâques 1067, Guillaume avait réuni à Fécamp une cour brillante. Toutefois, si l'abbaye tirait des bénéfices de l'union de la Normandie et de l'Angleterre, elle en subissait aussi les inconvénients, surtout pendant les luttes entre les fils de Guillaume de 1087 à 1094, puis entre Étienne de Blois et les Angevins.

Mots-clés : rois anglo-normands, Robert Courteheuse, frontières, le Précieux Sang, Première Croisade, justice ducale.

Abstract :

The experiences of the abbey of Fécamp can serve as an example of relations between the kingdukes and ecclesiastical magnates. The abbey had already acquired possessions in England before 1066 and assisted William's expedition. The Conqueror held a brilliant court at Fécamp at Easter 1067. Afterwards the periods when England was held together with Normandy brought some advantages, but there were also problems, especially during the struggles between the sons of the Conqueror and between Stephen of Blois and the Angevins.

Keywords: Anglo-Norman kings, Robert Curthose, frontiers, the Precious Blood, the First Crusade, ducal justice.

Ce colloque est pour moi l'occasion de replacer les expériences de l'abbaye de Fécamp dans le contexte des régimes des rois anglo-normands ${ }^{1}$. Je mettrai l'accent sur la période comprise entre 1066 et 1135, et me servirai de Fécamp pour mettre

1. Je voudrais remercier le Dr Evelyn Mullally qui a traduit cette communication en français. 
en lumière le fait selon lequel le règne intermittent des rois d'Angleterre a apporté aux normands autant de difficultés que d'avantages.

En 1066, les moines de Fécamp possédaient déjà, en Angleterre, des terres et des ports qui leur avaient été donnés par les rois d'Angleterre ${ }^{2}$. Ils s'occupèrent activement de l'expédition ducale car cette entreprise pouvait leur apporter la possibilité d'une protection supplémentaire pour leurs terres anglaises, la perspective d'autres gains et des promotions possibles, pour eux-mêmes, dans des abbayes et des évêchés d'Outre-Manche. La communauté avait les moyens de fournir à Guillaume des hommes et des vaissaux et pouvait aussi lui faire part de son expérience des traversées de la Manche et des ports anglais. Rémi l'Aumônier a affrété, dit-on, un vaisseau et fourni vingt chevaliers pour l'ost ducal ; un moine de Fécamp se trouvait même dans l'armée de Guillaume ${ }^{3}$. Le Conquérant a solennellement célébré Pâques à l'abbaye de Fécamp, en 1067. Ses vêtements somptueux et ceux des gens de sa cour ont forcé l'admiration ; les captifs aux cheveux longs attiraient aussi l'attention de son entourage ${ }^{4}$. Guillaume a confirmé et protégé les nombreuses terres de l'abbaye en Angleterre ${ }^{5}$. Des moines de Fécamp ont fait de belles carrières ecclésiastiques en Angleterre en devenant abbés et évêques : Rémi, à l'évêché de Dorchester (situé ensuite à Lincoln), Herbert Losinga, abbé à Ramsey puis évêque à Elmham (puis à Thetford et enfin à Norwich), Turold, abbé à Malmesbury puis à Peterborough et Vitalis, abbé à Westminster ${ }^{6}$.

Guillaume allait faire ses Pâques à l'abbaye au moins deux fois encore, en 1075 et en $1083^{7}$. Pourtant, comme l'a fait remarquer le Professeur Annie Renoux, l'élargissement de ses horizons a fait que Fécamp (tant l'abbaye que la résidence ducale voisine) devenait moins essentiel à ses yeux ainsi qu'à ceux de ses successeurs qu'il ne l'avait été à ses prédécesseurs à la fin du $\mathrm{X}^{\mathrm{e}}$ et au début du $\mathrm{XI}^{\mathrm{e}}$ siècle ${ }^{8}$. Après 1066 ,

2. Aethelred avait donné 'Rameslie'(un manoir qui comprenait Rye et Winchelsea) à Fécamp: HASKINS, Charles Homer, «A Charter of Canute for Fécamp », English Historical Review, 33, 1918, p. 342-324; le roi Edouard était le donateur du manoir de Steyning, qu'Harold Godwinson avait saisi, (BATES, David, Regesta..., n 141, 142); Domesday Book, I, 17. L'abbaye avait aussi des biens à Hastings, échangés pour le manoir de Bury après 1066 (BATES, Regesta...), nº 144. Les moines croyaient que le roi Edouard avait promis Bury à Fécamp (MABILLon, Jean, Annales Ordinis Sancti Benedicti, Paris, 1703-39, 4, p. 547b; voyez aussi Round, John Horace, "Some Early Sussex Charters », Sussex Archaeological Collections, 42, 1899, p. 75-86. Mark GARDINER a suggéré que la terre à Hastings était peut-être le site du château, «Shipping and Trade between England and the Continent during the Eleventh Century », Anglo-Norman Studies, 22, 1999, p. 91.

3. VAN HOUTS, Elisabeth M. C., "The Ship list of William the Conqueror ", Anglo-Norman Studies, 10, 1988, p. 178-179; Guillaume De Malmesbury, Gesta Pontificum, éd. N. E. S. A. Hamilton, Rolls Series, Londres, 1870, p. 312-313; The Gesta Guillelmi of William of Poitiers, éd. Ralph Henry Carless Davis et Marjorie ChibNall, Oxford, Clarendon Press (Oxford Medieval Texts), 1998, p. 120-121.

4. The Gesta Guillelmi..., p. 178-180

5. BATES, Regesta..., no $141-144$.

6. ChibNall, Marjorie, « Fécamp and England » in L’Abbaye bénédictine de Fécamp..., 1, p. 127-131.

7. The Ecclesiastical History of Orderic Vitalis, éd. Marjorie CHIBNALL, Oxford, Clarendon Press (Oxford Medieval Texts), 1969-80, t. 3, p. 8 [ci-après ORderic VITAL]; BATES, Regesta..., nº 230.

8. RENOUX, 1991, p. 492. 
il y avait beaucoup plus de communautés monastiques qui le pressaient de reconnaître leur droit à sa protection et qui demandaient des bienfaits supplémentaires. Le roi était toujours en voyage et peut-être n'avait-il pas le temps de visiter Fécamp.

Guillaume le Roux et Robert Courteheuse se sont battus pour y exercer leur suprématie entre 1088 et 1094 ; l'un et l'autre ont vraisembablement visité l'abbaye, à cette époque, mais ne l'ont plus visitée par la suite. Pour autant qu'on le sache, Henri I ${ }^{\text {er }}$ non plus ne s'y est pas rendu, car il préférait traverser la Manche pour se rendre en Angleterre en empruntant la route occidentale. Quand il se trouvait en Normandie, il avait tendance à tenir ses grandes cours à Rouen ou à Caen ${ }^{9}$. Fécamp avait été le lieu de sépulture des ducs Richard I ${ }^{\text {er }}$, Richard II ${ }^{10}$ et de Marguerite du Maine ${ }^{11}$, la fiancée de Robert Courteheuse, mais l'abbaye n'est pas devenue la nécropole de la maison régnante en Normandie comme, par exemple, Saint-Denis l'est devenue pour les Capétiens.

Les dernières années du XI ${ }^{e}$ siècle ont été difficiles pour l'abbaye, à cause des luttes entre les fils du Conquérant. Robert avait pourtant bien commencé à s'attirer les faveurs des moines en leur restituant certaines terres que l'abbaye avait perdues et il leur avait octroyé une foire qui devait durer tout le temps de la prise du hareng ${ }^{12}$. En revanche, dans ses efforts pour rassembler une flotte afin d'envahir l'Angleterre en 1088, il a capturé des vaisseaux appartenant aux moines; l'un d'eux, ancré dans la Seine, a été détourné de force ${ }^{13}$. Robert a tenté de renforcer son autorité aux environs de Fécamp, installant plusieurs de ses fidèles comme Guillaume Grenet, Gilbert Belet, Geoffroi Martel, Robert d'Estouteville, sur des terres issues du domaine ducal ${ }^{14}$. Pourtant, cette tentative a provoqué des troubles dans un cas au moins, et il a fait détruire le château d'un autre nouveau-venu, Robert de Mortagne ${ }^{15}$.

L'échec de son projet d'invasion fut si rapide que Robert lui-même ne s'est jamais embarqué pour l'Angleterre. Bientôt, il eut du mal à garder la fidélité de ses vassaux qui tenaient des terres près des frontières nord et nord-est de la Normandie. Parmi ceux-ci se trouvaient le comte d'Eu et le comte d'Aumale qui avaient tous

9. Christelow, Stephanie Mooers, «A Moveable Feast? Itineration and the Centralization of Government under Henry I », Journal of British Studies, 29, 1996, p. 206-7.

10. DUdOn De SAINT-QUENTIN, De moribus et actis primorum Normanniae ducum, éd. Jules LAIR, Mémoires de la Société des Antiquaires de Normandie, 23, Caen, 1865, p. 297-299; The Gesta Normannorum Ducum of William of Jumièges, Orderic Vitalis, and Robert of Torigni, éd. Elisabeth M. C. VAN Houts, 2 vol., Oxford, Clarendon Press (Oxford Medieval Texts), 1992, 1995, t. 2, p. 3840 [ci-après $G N D]$.

11. ORDERIC Vital, t. 2, p. 118.

12. HASKINS, Charles Homer, Norman Institutions, Cambridge, Cambridge Mass., Harvard University Press, 1918, p. 69.

13. Des miracles advenus en l'église de Fécamp, éd. Abbé Eugène-Paul SAUVAGE, Mélanges de la Société de l'Histoire de Normandie, $2^{e}$ série, 1893, p. 29-32.

14. LE MAHO, 1976, p. 58-59.

15. Pour l'identité de Robert de Bec-de-Mortagne voir AdIGARD DES GAUTRIES, Jean, « Les noms de communes en Normandie », Annales de Normandie, supplément (1962), p. 6, cité par LE MAHO, 1976, p. 56. 
deux des terres en Angleterre. D'autres seigneurs se sont laissés séduire par Guillaume le Roux ; on dit que Guillaume y a gagné toute la garnison à Saint-Valéry, ce qui allait lui fournir une tête de pont ${ }^{16}$. Le comte Etienne d'Aumale et Gérard de Gournay aussi passèrent à Guillaume le Roux et on pourrait en ajouter d'autres ${ }^{17}$. Quoiqu'il en soit, pendant l'été de 1089, Robert Courteheuse a assiégé les châteaux d'Eu et de La Ferté ${ }^{18}$.

Cependant, les moines de Fécamp sont entrés en conflit avec l'archevêque de Rouen, qui avait jeté l'interdit sur la Normandie, en réponse au duc Robert qui avait cédé le domaine archiépiscopal de Gisors au roi Philippe ${ }^{19}$. Les moines n'ont pas obéi, arguant du fait que leur abbé, Guillaume, se trouvait alors en Angleterre. Ils ont fait appel au pape en soulignant leur relation spéciale avec la papauté20. Par conséquent, les moines affaiblissaient ainsi leurs liens avec le duc, mais ils étaient prêts apparemment à accepter ce risque ${ }^{21}$.

C'est précisément au moment où l'abbaye mettait en avant son statut d'abbaye exempte qu'un programme de reconstruction fut commencé. L'abbé Guillaume pouvait financer de grands travaux de construction; il a allongé la nef et refait le choeur, fournissant ainsi un cadre pour la relique la plus révérée de l'abbaye, celle du Précieux Sang ${ }^{22}$. En même temps, on a révisé le dossier des miracles associés à cette relique, et Annie Renoux a suggéré que l'abbé Guillaume a conçu un "programme de substitution $»^{23}$. Plus que les liens étroits avec la dynastie ducale, l'abbaye s'affirmait comme un centre de pèlerinage. L'abbé Guillaume avait bien choisi son moment : il y avait une sensibilité accrue pour des reliques de ce genre dans les dernières années du XI ${ }^{e}$ siècle, l'époque de la Première Croisade ${ }^{24}$. Quand Baudry de Bourgueil a visité l'abbaye au début du XIIe siècle, il pouvait décrire l'abbaye comme un "palais de Dieu », parce qu'elle possédait le sang du Christ ${ }^{25}$.

Face aux révoltes soutenues par Guillaume le Roux, le duc Robert a décidé de négocier et a cédé l'abbaye de Fécamp, les comtés d'Eu et d'Aumale, le port de Cherbourg et l'abbaye du Mont-Saint-Michel à son frère ${ }^{26}$. Il y avait quelques autres

16. The Anglo-Saxon Chronicle, éd. Dorothy Whitelock, David C. Douglas et Susie I. Tucker, Londres, Eyre and Spottiswoode, 1961, p. 168.

17. Orderic Vital, t. 4, p. 182.

18. Regesta Regum Anglo-Normannorum, éd. Henry William Carless DAVIS, t. 1, Oxford, 1913, n 310; Gallia Christiana in provinciis ecclesiasticis distributa, 11, Paris, 1759, instr. 18; BARLOW, 1983, p. 272-273.

19. Gallia Christiana, t. 11, instr. 18; BARLOW, 1983, p. 273.

20. LEMARIGNIER, 1937, p. 192-204.

21. RENOUX, 1991, p. 493-495.

22. ORDERIC Vital, t. 6, p. 128.

23. RENOUX, 1991, p. 496-498.

24. Herval, René, "En Marge de la légende du Précieux Sang - Lucques - Fécamp - Glastonbury », in L'Abbaye bénédictine de Fécamp..., t. 1, p. 105-126.

25. Baudry of Bourgueil, Opera, éd. Jacques-Paul Migne, Patrologia Latina, 166, col. 1176, 1182.

26. Orderic Vital, t. 4, p. 236, 250; GND, 2, p. 206-207; Anglo-Saxon Chronicle, 1091; The Chronicle of John of Worcester, éd. Patrick MC GURK, Oxford, Clarendon Press (Oxford Medieval Texts), t. 3, 1998, p. 58 [ci-après JEAN DE WORCESTER]. 
propositions : le roi promit d'aider le duc à soumettre les châteaux normands et le comté du Maine; de restituer aux partisans du duc leurs propriétés anglaises et de donner au duc lui-même des propriétés anglaises. Le duc et le roi ont promis que chacun serait le successeur de l'autre à défaut d'un héritier légitime.

Mais l'accord n'était pas viable et le duc a condamné son frère parce qu'il n'avait pas tenu ses promesses. Guillaume le Roux est arrivé en Normandie et les frères ont discuté de leurs différends, mais sans résultat ${ }^{27}$. Guillaume assiéga le château de Bures-en-Bray alors que le duc avec son suzerain, le roi Philippe, attaquait le château d'Argentan au sud de la Normandie ${ }^{28}$. Le roi Guillaume rentra en Angleterre pour lever des hommes et de l'argent, alors que le duc et son allié le roi Philippe avançaient vers Eu. Les richesses du roi d'Angleterre furent décisives : le roi Philippe abandonna la lutte.

Le duc Robert avait eu des problèmes avec les seigneurs du nord du duché ; de son côté, le roi Guillaume eut les siens en Angleterre, l'année suivante, lors d'une grande révolte dont deux des chefs étaient Guillaume, comte d'Eu et Etienne, comte d'Aumale ${ }^{29}$. Il a envoyé son frère Henri Beauclerc en Normandie à sa place, mais on ne sait pas où. À ce moment, le pape a prêché la Première Croisade ; le duc lui-même et quelques seigneurs du voisinage de Fécamp ont quitté le duché $^{30}$.

À Fécamp, la reconstruction de l'église a continué. La date de la consécration n'est pas assurée (1099 ou 1106), mais 1099 est toutefois plus probable parce que le duc n'y assista pas $^{31}$. Néanmoins l'abbé Guillaume a réussi à obtenir de Guillaume le Roux la protection des droits de l'abbaye à Steyning, en Angleterre. Là-bas, la trop grande proximité d'un nouveau seigneur normand, Philippe de Briouze, et d'une communauté de moines à Bramber, qui venaient de Saint-Florent-de-Saumur, étaient la cause d'un désaccord persistant ${ }^{32}$.

Quand les Normands sont rentrés de Jérusalem, Henri Beauclerc avait succédé à Guillaume le Roux, comme roi d'Angleterre. Robert Courteheuse s'est donc trouvé écarté du trône pour la deuxième fois. Une fois de plus, Robert a rassemblé ses forces pour une invasion, et cette fois, sa flotte a pris la mer au Tréport ${ }^{33}$. Nous ignorons la réaction des moines de Fécamp à ces événements. Pourtant, en 1103, l'abbé Guillaume se trouvait en Angleterre, où il avait obtenu une audience au sujet de la dispute prolongée entre Fécamp et, d'autre part, Philippe de Briouze et les

27. JEAN DE WORCESTER, t. 3, p. 70-71.

28. JEAN DE WORCESTER, t. 3, p. 70-73.

29. Anglo-Saxon Chronicle, éd. Whitelock, Douglas et TuCKer, p. 172-173; JeAn DE WorCester, t. 3, p. 76-78.

30. DAVID, Charles Wendell, Robert Curthose, duke of Normandy, Cambridge, MA, 1920, appendix D: Robert's Companions on the Crusade.

31. RENOUX, 1991, p. 503.

32. VAN CAENEGEM, Ralph C., English Lawsuits from William I to Richard I, 1, William I to Stephen, Selden Society, 106, 1990, nº 163

33. JEAN DE WORCESTER, 3, p. 96. 
moines de Bramber. Cette fois, il semble que le jugement a été décisif, puisqu'on n'entendit plus parler de ces problèmes. L'autorité du duc s'est relativement maintenue en haute Normandie. Robert d'Estouteville en particulier s'est montré un allié à toute épreuve ${ }^{34}$. Ce n'est que trois ans plus tard qu'Henri a vaincu et capturé son frère Robert à la bataille de Tinchebray, dont un prêtre de Fécamp avait été un témoin oculaire ${ }^{35}$.

En mars 1107, l'abbé Guillaume a assisté au conseil d'Henri à Lisieu $x^{6}$ ? Cette assemblée faisait partie d'une série de conseils où Henri a exposé les principes selon lesquels il se proposait d'opérer en Normandie. Selon Orderic Vital, ces principes comportaient une proclamation de paix, la suppression du vol et du pillage, ainsi que la restauration du domaine ducal et des terres ecclésiastiques tels qu'ils se trouvaient à la mort de son père le Conquérant ${ }^{37}$. En plus, Henri a mandé ceux qui avaient des pouvoirs de justice et de juridiction et, en somme, il leur a donné des admonestations vigoureuses ${ }^{38}$. Henri avait justifié l'invasion de la Normandie en invoquant l'incapacité de son frère Robert à protéger l'église et le peuple ${ }^{39}$; lui, au contraire, prenait très au sérieux son rôle de protecteur de l'église, comme les moines de Fécamp allaient s'en rendre compte.

La promptitude de l'abbé Guillaume à se rendre à la cour d'Henri a bientôt profité à Fécamp, car le roi a présidé un procès entre les moines de cette abbaye et ceux de Saint-Taurin d'Evreux, qui cherchaient à échapper à la tutelle de Fécamp. Devant Henri, leur tentative d'émancipation a échouée ${ }^{40}$.

L'abbé Guillaume était mort au cours de son retour du concile de Lisieux, et il fallait élire son successeur ${ }^{41}$. Le choix des moines s'est porté sur Roger de Bayeux qui avait été, paraît-il, très ami avec Guillaume, mais qui avait moins d'envergure que son prédécesseur ${ }^{42}$. La décision relevait essentiellement du pouvoir d'Henri, mais, comme Roger avait été l'ami de Guillaume, Henri a pu se montrer conciliant pour ne pas contrarier les moines.

A un moment inconnu, Henri avait pris le pouvoir au château de Fécamp, dont la garnison était restée fidèle même durant la guerre, en $1118^{43}$. Il devait s'efforcer ensuite d'établir son autorité sur les nobles des environs. Le temps et le hasard faisaient leurs effets : Gautier Giffard ${ }^{44}$ et Gérard de Gournay étaient morts ne

34. ORDERIC Vital, t. 6, p. 72, 84, 90.

35. DAVIS, Henry William Carless, "A Contemporary Account of the Battle of Tinchebray », English Historical Review, 24, 1909, p. 728-733.

36. ORDERIC VITAL, t. 6, p. 138.

37. ORderic Vital, t. 6, p. 92-94.

38. ORDERIC Vital, t. 6, p. 136.

39. Orderic Vital, t. 6, p. 86.

40. Regesta, éd. JOHNSON et Cronne, t. 2, nº 790

41. ORDERIC Vital, t. 6, p. 138-40.

42. Si l'on se réfere au propos d'Adelelm à Baudry de Bourgueil, éd. Jacques-Paul MigNE, Patrologia Latina, 166, col. 1177, cité par ORDERIC VITAL, 6, p. 140-141.

43. ORDERIC VITAL, t. 6, p. 222

44. ORDERIC VitAL, t. 6, p. 38. 
laissant que des fils encore mineurs ${ }^{45}$. Henri s'en conciliait d'autres en leur donnant des terres, comme à Guillaume Martel, qui a attesté un document royal pour la première fois vers 1128 ; il a aussi attesté chacune des deux interventions en faveur de Fécamp en $1131^{46}$.

Henri n'arrivait pas, pourtant, à se concilier tous les Normands. Les d'Estouteville, par exemple, n'avaient pas perdu leurs terres normandes en 1106, mais ils ne parvinrent pas pour autant à la cour d'Henri. Plusieurs de ces seigneurs qui avaient causé des ennuis à Robert Courteheuse et à Guillaume le Roux allaient se révolter contre Henri dès que les circonstances le permirent. En 1118, des combats sérieux eurent lieu dans les pays de Talou et de Caux, et cette révolte avait pour chefs une alliance des seigneurs voisins, y compris Etienne comte d'Aumale, Henri comte d'Eu, et Gérard de Fécamp ; le jeune Hugues de Gournay en fit partie dès qu'il eut atteint sa majorité ${ }^{47}$. Par contre, la garnison de Fécamp restait fidèle, et ce succès illustre bien un aspect clé du régime d'Henri en Normandie : il a pris le contrôle de châteaux et il a dépensé des sommes importantes pour les construire et les garnir : c'est ainsi qu'il a pu traverser cette année si périlleuse pour lui.

Il n'est pas facile de rassembler les indices qui permettent d'éclairer les caractéristiques du gouvernement d'Henri dans cette région. Qu'est devenue la vicomté de Fécamp? Si, comme M. Bouvris le suggère ${ }^{48}$, le rebelle Gérard de Fécamp sortait du lignage qui avait tenu auparavant l'office de vicomte, il est alors possible qu'Henri ait transféré la vicomté à l'abbé, car plus tard, en 1180, cet office était effectivement tenu par l'abbé de Fécamp ${ }^{49}$. De toute façon, l'abbé de Fécamp a conservé la faveur d'Henri. On note la présence de l'abbé en Angleterre en $1115^{50}$. En outre, il a assisté à des conciles ecclésiastiques à Rouen en 1115 et $1128^{51}$. Lors de ce dernier concile, Henri a protégé, dit-on, les droits des abbayes contre les évêques et les archevêques.

L'abbaye avait d'importants pouvoirs de haute justice, mais nous entendons parler aussi des juges d'Henri, qui avaient le droit d'entendre certains plaids. Il est même devenu nécessaire d'ordonner aux officiers du roi de donner à l'abbé sa part légitime des amendes qui provenaient des cas d'incendie criminel ou d'homicide ${ }^{52}$. On décrit aussi l'administration des droits de chasse du duc dans la forêt de Fécamp et, là encore, il fallait obliger les officiers d'Henri à donner aux moines les

45. ORDERIC VITAL, t. 6, p. 190.

46. Regesta, éd. JohnSOn et Cronne, t. 2, nº 1562, 1689, 1690.

47. ORderic Vital, t. 6, p. 190-194.

48. BouvrIS, Jean-Michel, "Contribution à une étude de l'institution vicomtale en Normandie au $\mathrm{XI}^{\mathrm{e}}$ siècle. L'exemple de la partie orientale du duché: les vicomtes de Rouen et de Fécamp ", Autour du pouvoir ducal normand $X^{e}-X I I^{e}$ siècles, Cahier des Annales de Normandie $\mathrm{n}^{\circ}$ 17, 1985, p. 173.

49. Magni Rotuli Scaccarii Normanniae sub regibus Angliae, éd. Thomas STAPLETON, Londres, Société des Antiquaires de Londres, 1840-1844, t. 1, p. 90. Une autre possibilité est que Guillaume Martel tenait le château et vicomté pendant les dernières années du règne.

50. Regesta, éd. Johnson et Cronne, t. 2, n 1092.

51. Orderic Vital, t. 6, p. 202, 388-390.

52. Regesta, éd. JOHNSON et CRONNE, t. $2, \mathrm{n}^{\circ} 1562$. 
dîmes de la chasse qui leur étaient dues ${ }^{53}$. En Angleterre, aussi, les moines de Fécamp devaient veiller sur leurs intérêts. Ils se sont disputés avec le comte d'Eu au sujet de leurs droits importants sur les péages des ports de Winchelsea et de Hastings. L'accord s'est fait dans la cour du roi, mais au prix fort : le roi a obtenu une somme considérable pour son intervention aussi bien que sa part des péages ${ }^{54}$. La paix était établie, mais il fallait la payer. Nous pouvons noter aussi que les interventions d'Henri au sujet des amendes judiciaires et des droits de chasse se sont produites à un moment où il se montrait particulièrement généreux dans ses dons à l'église pour des raisons tant dynastiques que spirituelles ${ }^{55}$. Il faut dire, pourtant, qu'Henri était prêt à reconnaître son devoir de protéger l'abbaye.

Henri n'a pas accordé à Fécamp de nouvelles terres ou de nouveaux privilèges, mais l'abbaye était déjà très riche et, à cet égard, les relations du roi avec Fécamp peuvent se comparer avec celles qu'il a eues avec Le Mont-Saint-Michel ou, en Angleterre, avec l'abbaye de Westminster ${ }^{56}$. Ses préférences personnelles, comme on sait, l'attiraient vers Cluny, et en Normandie, vers les moines du Bec. Il avait aussi des raisons tant politiques que spirituelles pour protéger le nouvel ordre de Savigny, qui se situait près de la frontière entre la Normandie et le Maine. Il en était de même pour sa générosité envers la maison angevine de Fontevraud et les Templiers, à la suite du mariage de sa fille avec Geoffroi d'Anjou ${ }^{57}$. Comme Mathieu Arnoux l'a montré, Henri a joué un rôle considérable dans la fondation des maisons d'Augustins des deux côtés de la Manche ${ }^{58}$. Vers la fin de sa vie, il soutenait les Cisterciens ${ }^{59}$. Il est vrai que sa façon de traiter les maisons bien établies de Bénédictins, en Angleterre comme en Normandie, était beaucoup moins éclatante, mais il ne faut pas croire qu'il les négligeait; au contraire, sa protection restait active ; elle constituait une partie importante de son action politique.

53. Regesta, éd. JohnSON et Cronne, t. 2, $\mathrm{n}^{\circ} 1689$.

54. Regesta, éd. Johnson et Cronne, t. 2, n 1690; Magnum Rotulum Scaccarii vel Magnum Rotulum Pipae de Anno Tricesimo-Primo Regni Henrici Primi, éd. Joseph HunTER, Londres, 1833, p. 71.

55. Voyez Judith GrEEN, « Piety and Patronage of Henry I », Haskins Society Journal, à paraître.

56. Il semble qu'Henri ${ }^{\text {er }}$ n'a pas fait des nouveaux dons au Mont-Saint-Michel: deux textes seulement sont inclus au catalogue de ses actes, Regesta, éd. JOHNSON et CRONNE, t. 2, nº 1418 (un échange pour les églises de Wargrave et Cholsey), 1422 (la soumission de Thomas de Saint-Jean). Pour Westminster voyez: MASON, Emma, «Westminster Abbey and the Monarchy between the reign of William I and John », Journal of Ecclesiastical History, 41, 1990, p. 209-210.

57. Fontevraud: Regesta, éd. JOHNSON et CRONNE, t. 2, nº 1580-1, 1687; Templiers: GND, 2, p. 256257.

58. ARnOuX, Mathieu (dir.), Des clercs au service de la réforme. Etudes et documents sur les chanoines réguliers de la province de Rouen, Bibliotheca Victorina, 11, Turnhout, Brepols, 2000, p. 120, 142144, 215-220; DiCKINSON, John Compton, The Origins of the Austin Canons and their introduction into England, Londres, 1950.

59. Le premier cartulaire de l'abbaye cistercienne de Pontigny (XII ${ }^{e}$-XIII ${ }^{e}$ siècles), éd. Michel GARRIGUES, Paris, 1981, p. 86; Mortemer: BouvET, J., "Le récit de la fondation de Mortemer », Collectanea Ordinis Cisterciensium Reformatorum, 22, 1960, p. 149-168; Rievaulx: Regesta, éd. JOHNSON et Cronne, t. 2, n 1720, 1740-1, 1961. 
Henri pacisque serenus amator: C'est comme cela que le moine Orderic Vital a caractérisé ce roi ${ }^{60}$, et c'est comme roi pacificateur qu'on s'est souvenu de lui à Fécamp, comme en Angleterre. Son régime était autoritaire, pour ne pas dire plus, mais pour les moines, ce régime valait mieux que le désordre qui le suivrait, selon l'abbé Henri de Sully, neveu du roi Etienne, élu en 1140, le vieux roi Henri avait protégé les intérêts de l'abbaye des deux côtés de la Manche ${ }^{61}$. Quand Geoffroi d'Anjou et son fils ont maîtrisé le duché, ils ont confirmé les terres et les privilèges de l'abbaye ${ }^{62}$. En Angleterre, il y avait des difficultés dans l'Oxfordshire, un pays férocement disputé, où l'abbaye possédait un petit prieuré à Cogges (maintenant un quartier de Witney, non loin d'Oxford). Un moine de Fécamp visita le prieuré et il essaya de percevoir des rentes et des dîmes dans le Kent. Ce récit n'est pas daté, mais paraît avoir été écrit dans les années 1150. Il révèle toute une série de problèmes, dûs à la nature et à l'homme ${ }^{63}$. Une lettre de l'archevêque Theobald, dans sa capacité de légat papal, confirme son témoignage ${ }^{64}$.

La conquête de l'Angleterre avait donc apporté des avantages et des inconvénients à Fécamp. Pour les moines, il valait mieux que la Normandie et l'Angleterre soient tenues par un seul homme. Des difficultés avaient surgi essentiellement à cause de successions disputées. Les disputes les plus longues pour l'abbaye étaient les conflits locaux, notamment entre 1088 et 1094 et aussi en 1118. Dans une certaine mesure, Henri Ier d'Angleterre avait rétabli l'ordre. Il utilisait ses richesses anglaises et sa force militaire pour concilier, pour dominer et pour punir les rebelles. La paix, vraiment, mais un certain genre de paix.

\section{Bibliographie}

L'Abbaye bénédictine de Fécamp. Ouvrage scientifique du XIII centenaire 6581958, 4 vol., Fécamp, L. Durand et fils, 1959-63, XX + 415 p., 309 p., 167 p., $391 \mathrm{p}$.

BARLOW, Frank, William Rufus, Londres, Methuen, 1983, XIX + 484 p.

BATES, David, Regesta Regum Anglo-Normannorum. The Acta of William I (1066-1087), éd. Oxford, Clarendon Press, 1998, XXXVIII + 1153 p.

60. ORderic Vital, t. 6, p. 452.

61. Voyez la lettre de l'abbé Henri de Sully qui met en contraste la paix du régime d'Henri I ${ }^{\text {er }}$ avec les "multiplices et maximas perpessa... molestias" après sa mort, "Epistolae Fiscannenses, Lettres d'amitié, de gouvernement et d'affaires (XI-XII siècles) », éd. Jean LAPORTE, Revue Mabillon, 42, 1953, p. 26-27.

62. Regesta Regum Anglo-Normannorum, éd. Henry Alfred CRONNE and Ralph Henry Carless DAVIS, t. 3, Oxford, Oxford Clarendon Press, 1968, n 304, 305.

63. «Epistolae Fiscannenses", éd. LAPORTE, p. 29-31; la date du texte est révisée par John BLAIR, «Investigations at Cogges Priory, Oxfordshire, 1978-1981: the priory and parish church », Oxoniensia, 47, 1982, p. 47-48.

64. Theobald, Archbishop of Canterbury, éd. Avrom SALTMan, Londres, 1956, p. 330-331. 
The Ecclesiastical History of Orderic Vitalis, éd. Marjorie CHIBNALL, 6 vol., Oxford, Clarendon Press (Oxford Medieval Texts), 1969-1980.

Des miracles advenus en l'église de Fécamp, éd. Abbé Eugène-Paul SAUVAGE, Mélanges de la Société de l'Histoire de Normandie, 2e série, 1893, p. 9-49.

LE MAHO, Jacques, «L'apparition des seigneuries châtelaines dans le GrandCaux à l'époque ducale » Archéologie Médiévale, 6, 1976, p. 5-148.

LEMARIGNIER, Jean-François, Etude sur les privilèges d'exemption et de juridiction ecclésiastique des abbayes normandes des origines à 1140, Paris, A. PICARD, 1937, XXXIII $+331 \mathrm{p}$.

The Chronicle of John of Worcester, éd. Patrick MC GURK, vol. 3, Oxford, Clarendon Press (Oxford Medieval Texts), 1998, I + 351 p.

Migne, Jacques-Paul, Patrologiae cursus completus. Series Latina, 221 vol., Paris, 1844-1865.

Regesta Regum Anglo-Normannorum 1066-1154, 4 vol., vol. 1, éd. Henry William Carless DAvIs; vol. 2, éd. Charles, JoHnson and Henry Alfred, Cronne; vol. 3 et 4, éd. Henry Alfred Cronne, and Ralph Henry Carless DAVIS, Oxford, Clarendon Press (Oxford Medieval Texts), 1913-1969.

Renoux, Annie, Fécamp. Du palais ducal au palais de Dieu. Bilan historique et archéologique des recherches menées sur le site du château des ducs de Normandie (II siècle A. C. - XVII siècle P. C.), Paris, CNRS, 1991, 734 p.

The Gesta Normannorum Ducum of William of Jumièges, Orderic Vitalis, and Robert of Torigni, éd. Elisabeth M. C., VAN HouTs, 2 vol., Oxford, Clarendon Press (Oxford Medieval Texts), 1992 (CXXXIII + 156 p.), 1995 (XV + 341 p.). 